\title{
Effect of Alkyl Chain Length and Unsaturation of the Phospholipid on the Physicochemical Properties of Lipid Nanoparticles
}

\author{
Yuka Funakoshi, Yasunori Iwao, Shuji Noguchi, and Shigeru Itai* \\ Department of Pharmaceutical Engineering, Graduate School of Pharmaceutical Sciences, University of Shizuoka; \\ 52-1 Yada, Suruga-ku, Shizuoka 422-8526, Japan. \\ Received January 22, 2015; accepted June 11, 2015
}

Previously, we developed lipid nanoparticles (LNs) containing poorly water-soluble drugs using two types of phospholipids, a neutral phospholipid (hydrogenated soybean phosphatidylcholine) and a negativelycharged phospholipid (dipalmitoylphosphatidylglycerol), with mean particle sizes of less than $100 \mathrm{~nm}$. Here, we studied the effects of alkyl chain length and unsaturation of neutral and negatively-charged phospholipids on the physicochemical properties of LNs. Three neutral phospholipids, dimyristoylphosphatidylcholine, dipalmitoylphosphatidylcholine and distearoylphosphatidylcholine, having different alkyl chain lengths, were compared. The mean particle size of the LNs increased with the alkyl chain length, while the concentration of the drug entrapped in the LNs decreased. The particle size of all of the LNs could be maintained at less than $100 \mathrm{~nm}$ for 1 month in cool and dark conditions, with the LNs with longer alkyl chain lipids showing greater stability. In the unsaturated phospholipids, the double bond in the alkyl chain of dioleoylphosphatidylcholine and dierucoylphosphatidylcholine did not affect the physicochemical properties of the LNs. The negatively-charged phospholipids dipalmitoylphosphatidylglycerol and distearoylphosphatidylglycerol were also compared; LNs with longer alkyl chain lipids had larger particle sizes and lower drug concentrations, similar to the results for neutral phospholipids. We concluded that although some changes in physicochemical properties were observed among LNs with different phospholipid alkyl chain lengths, this methodology was general. LNs with suitable physicochemical properties could be prepared irrespective of the type of phospholipids used.

Key words lipid nanoparticle; poorly water-soluble drug; phospholipid; alkyl chain

Many candidate active pharmaceutical ingredients (APIs) in formulations show poor solubility in water. To enhance the solubility of such poorly water-soluble APIs, the use of nanoparticle formulations with particle sizes less than $100 \mathrm{~nm}$ has recently attracted considerable attention in the field of pharmaceutical research. ${ }^{1-3)}$ Recently, we successfully prepared lipid nanoparticles (LNs), which had a mean particle size of approximately $50 \mathrm{~nm}$ with a narrow particle size distribution, using wet milling, roll mixing, and high pressure homogenization to form small particles. ${ }^{4}$ The LNs improved the solubility of nifedipine (NI), a poorly water-soluble drug, and improved its oral absorption of NI when NI-LNs were administrated to rats. ${ }^{5)}$ NI-LNs showed excellent long-term stability in suspension for approximately 4 months in cool and dark conditions, and freeze-drying techniques combined with sugar as a cryoprotectant allowed the preparation of LNs with a good aqueous re-dispersibility. ${ }^{6,7)}$ NI-LNs lyophilized with trehalose exhibited suitable pharmacokinetic properties and good biocompatibility. ${ }^{8)}$ Generally, two types of phospholipids, neutral phosphatidylcholine (PC) and negatively-charged phosphatidylglycerol (PG) are used to prepare LNs. PG was added to the LNs to improve the dispersibility because of electrostatic repulsion. Here, we described the effect of the alkyl chain length and level of saturation of PC and PG on the physicochemical properties of the LNs using various kinds of phospholipids (Fig. 1).

\section{Results and Discussion}

Effect of Alkyl Chain Length of PC We prepared LNs consisting of dimyristoylphosphatidylcholine (DMPC)/dipal- mitoylphosphatidylglycerol (DPPG), dipalmitoylphosphatidylcholine (DPPC)/DPPG, and distearoylphosphatidylcholine (DSPC)/DPPG, which had mean particle sizes of 46, 53, and $61 \mathrm{~nm}$, respectively (Fig. 2A). The mean particle sizes tended to increase as the alkyl chain length of the phospholipid increased. The values of zeta potential of LNs using DMPC, DPPC, and DSPC were $-50,-51$, and $-50 \mathrm{mV}$, respectively (Fig. 2B) and there were no significant difference, indicating no significant relationships between zeta potential and particle size or alkyl chain lengths. The drug concentrations in LN suspensions using DMPC, DPPC, and DSPC were 106, 77.8, and $71.6 \mu \mathrm{g} \mathrm{mL}^{-1}$, respectively (Fig. 2C) and a 4-5-fold increase compared with the NI intact powder $\left(19.5 \mu \mathrm{g} \mathrm{mL}^{-1}\right){ }^{9}{ }^{9}$ The calculated entrapment efficiencies for DMPC, DPPC, and DSPC LNs were 99.5, 98.9, and 98.6\%, respectively and these values were similar to that of LNs with HSPC (99.5\%), suggesting that almost all the NI was found to be entrapped in the LNs. However, the drug concentration decreased as the alkyl chain length of the phospholipid increased. Next, the storage stability of the prepared LNs after 1 month in cool $\left(4^{\circ} \mathrm{C}\right)$ and dark conditions is shown in Fig. 3. LNs with DMPC showed a $30 \%$ increase in mean particle size compared with the size just after preparation. In contrast, the mean particle size of LNs with DPPC and DSPC increased by only $14 \%$ and $4 \%$, respectively (Fig. 3A). There were no significant changes, before and after storage. The drug concentrations in LN suspensions were 52.3, 74.7, and $60.7 \mu \mathrm{g} \mathrm{mL}^{-1}$ for DMPC, DPPC, and DSPC, respectively, 1 month after preparation (Fig. 3B). LNs with DMPC showed a $51 \%$ decrease in drug concentrations compared with just after preparation. The drug concentration 


\section{HSPC}

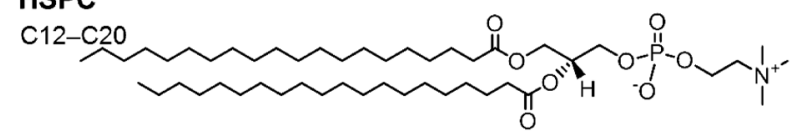

DMPC

C14:0/14:0

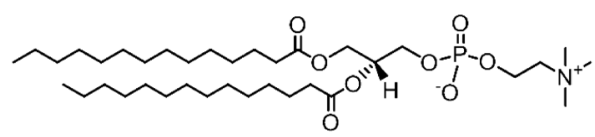

DPPC

C16:0/16:0

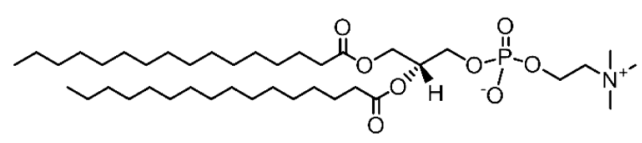

DSPC

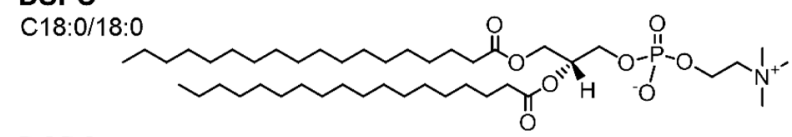

DOPC

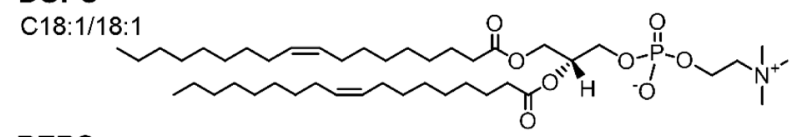

DEPC

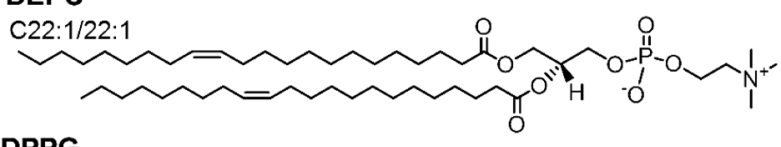

DPPG

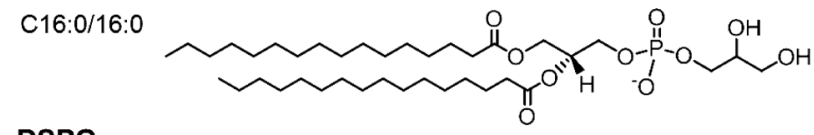

DSPG

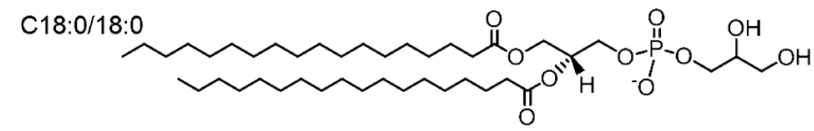

Fig. 1. Chemical Structures of the Drug Carriers

in the LN suspensions with DPPC and DSPC was maintained for 1 month.

The particle size of the LNs tended to increase, and the drug concentration in LN suspensions tended to decrease, with increasing alkyl chain length. This tendency of particle size changes conforms to previous reports on liposomes. ${ }^{10,11)}$ Due to the larger particle size of the LNs with longer alkyl chain lipids, these particles could not pass through the filter and a decrease in drug concentration might be observed. However, our group recently reported that when LNs with a mean particle sizes of 68.5 and $93.3 \mathrm{~nm}$ were orally administrated to rats, oral absorption of NI was improved in both samples. ${ }^{8}$ Therefore, we consider that the differences in mean particle size observed in this study would not adversely affect the pharmacokinetics.

LNs with DMPC were unstable 1 month after preparation; this phenomenon might be attributed to fast aggregation of the LNs or the weak drug entrapment ability of the DMPC LNs. DMPC LNs showed a narrow particle size distribution with a large amount of small particles (left part of Fig. 4A) compared with DSPC LNs (left part of Fig. 4B) just after preparation. The smaller LNs were thermodynamically unstable and may easily adsorb to larger LNs in suspension by Ostwald ripening. ${ }^{12)}$ In fact, DMPC LNs featured a biphasic size distribution with peaks at 30 and $110 \mathrm{~nm}$ (right part of Fig. 4A), while DSPC LNs 1 month after preparation showed a single peak (right part of Fig. 4B). In addition, the polydispersion index of
A)

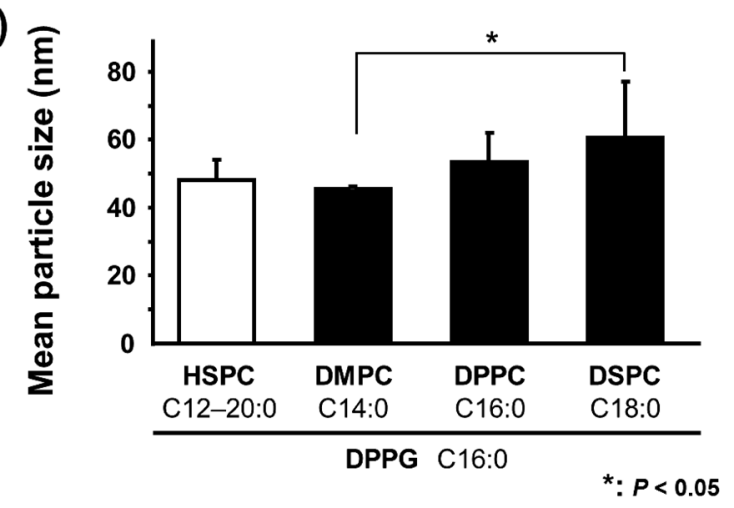

B)

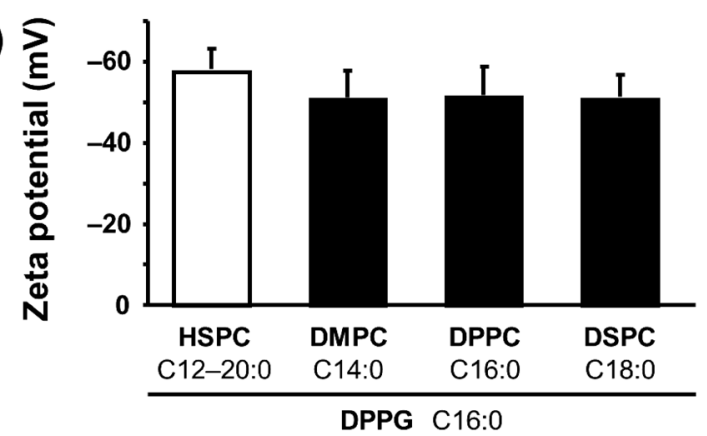

C)

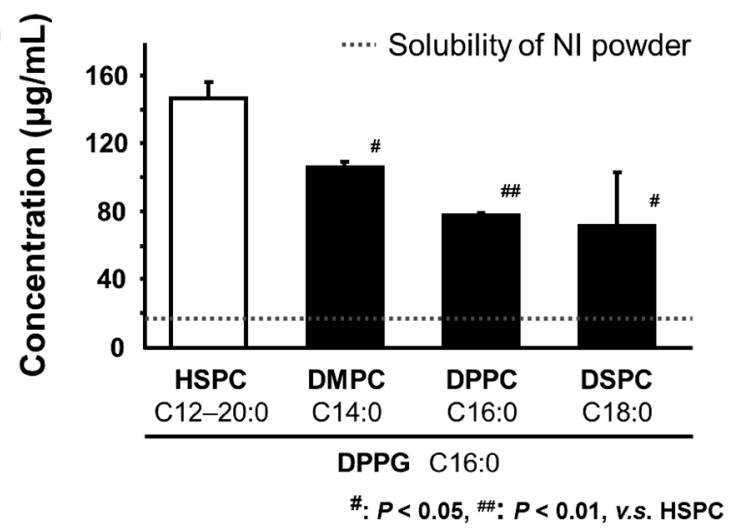

Fig. 2. Effect of Alkyl Chain Length of PC on LNs Containing DPPG (C16:0)

(A) Mean particle size, (B) zeta potential and (C) concentration of NI. These data are the average values obtained from three experiments $( \pm$ S.D.).

LNs with DMPC increased from 0.19 to 0.28 . The size distribution of DPPC LNs was similar to that of DSPC LNs. Therefore, the smaller particles found in DMPC LNs were involved in the fast aggregation of LNs resulting in the increased particle size. In addition, because of this aggregation, some change in the lipid layer may have occurred leading to leakage of the entrapped drug from the LNs. A yellow precipitate was observed in the bottom of the storage beaker and was assumed to be leaked NI (data not shown). Previous reports have demonstrated that drug entrapment ability and bilayer stability in liposomes were enhanced when longer alkyl chain lipids were used, as they formed a more rigid lipid membrane owing to van der Waals forces. ${ }^{10,11)}$ This suggests that the weak drug entrapment ability of DMPC LN may also be involved in the decrease in drug concentration under storage conditions. Overall, these data indicate that although the particle sizes of LNs are increased to some extent, the use of longer alkyl chain lipids might provide LNs with good stability. 


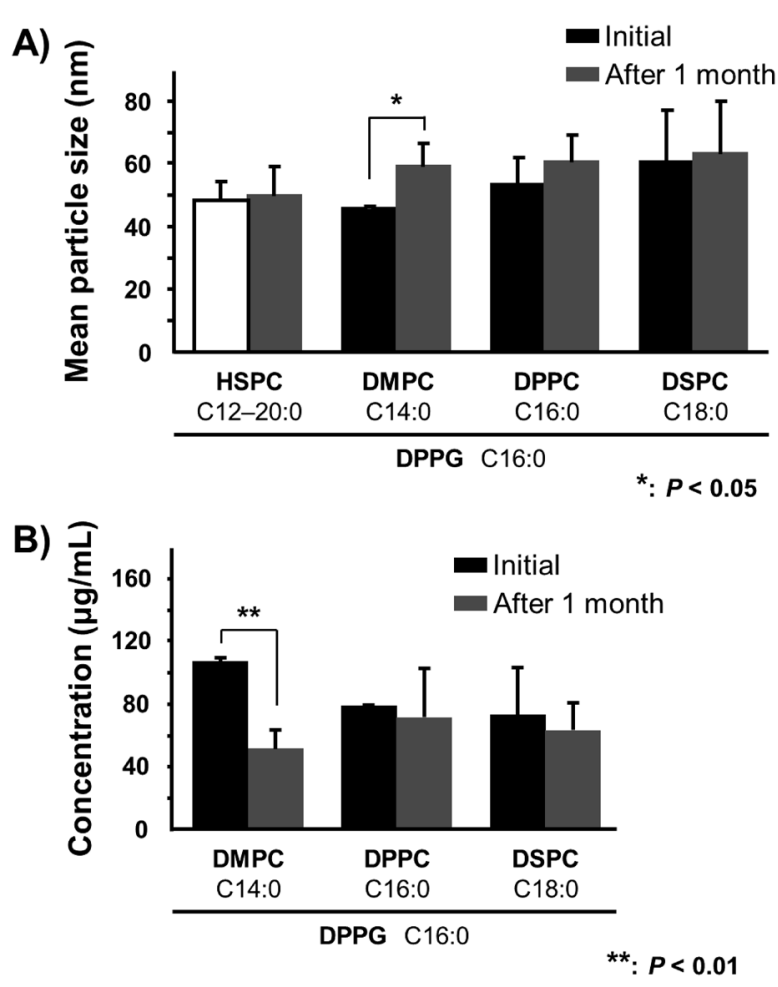

Fig. 3. Stability of LN Suspensions

(A) Mean particle size and (B) concentration of NI. These data are the average values obtained from three experiments $( \pm$ S.D.).
Effect of an Unsaturated Bond in PC Next, we prepared LNs consisting of dioleoylphosphatidylcholine (DOPC)/ DPPG and dierucoylphosphatidylcholine (DEPC)/DPPG. LNs prepared using DOPC and DEPC, which have an unsaturated bond in the alkyl chain, had mean particle sizes of 57 and $59 \mathrm{~nm}$, respectively (Fig. 5A). There were no significant differences in the mean particle size of LNs with shorter DOPC $(18: 1)$ or longer DEPC $(22: 1)$ chain lengths. Zeta potential of LNs using DOPC and DEPC were -60 and $-81 \mathrm{mV}$, respectively (Fig. 5B). The drug concentration of each LN was determined (Fig. 5C) and both LNs had a high drug entrapment efficiency of $99.8 \%$. The drug concentrations in LN suspensions using DOPC and DEPC were 108 and $126 \mu \mathrm{g} \mathrm{mL}^{-1}$, respectively (Fig. 5C) indicating at least a 5-fold increase compared with the NI intact powder. There were no significant differences. The drug concentration in both the unsaturated LN suspensions was approximately $70-90 \%$ of the concentration in LNs with HSPC. When the LNs were stored in cool and dark conditions for 1 month, LNs with DOPC and DEPC had mean particle sizes of 65 and $61 \mathrm{~nm}$, respectively. There were no significant changes compared with just after preparation (Fig. 6).

In LNs prepared using saturated DSPC (Fig. 2A) and unsaturated DOPC (Fig. 5A), which have the same number of carbons in the alkyl chains, no significant difference in the mean particle size was observed. Therefore, we suggest that the presence of an unsaturated bond in the alkyl chain of PC has no effect on the mean particle size of LNs used in this study. Additionally, the tendency towards similar particle sizes for LNs with DOPC and DEPC, which have different alkyl chains lengths, (Fig. 5A) might be explained by the LNs having the same degree of unsaturation. Unsaturated phospholipids may

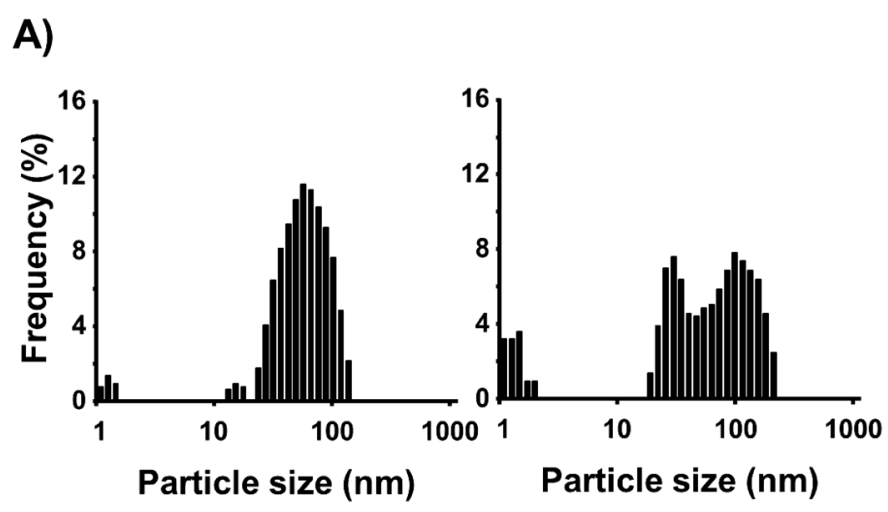

B)

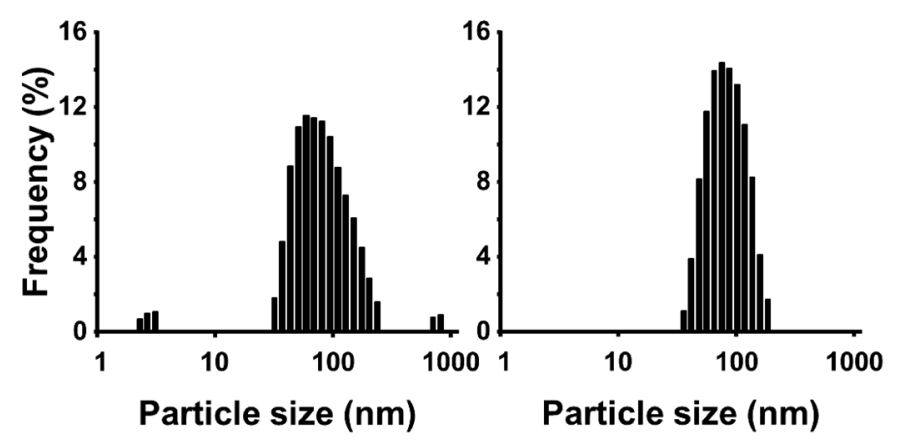

Fig. 4. Particle Size Distribution of LNs

(A) LNs prepared using DMPC and DPPG and (B) LNs prepared using DSPC and DPPG. The left figure shows just after preparation and the right figure shows 1 month after preparation. 

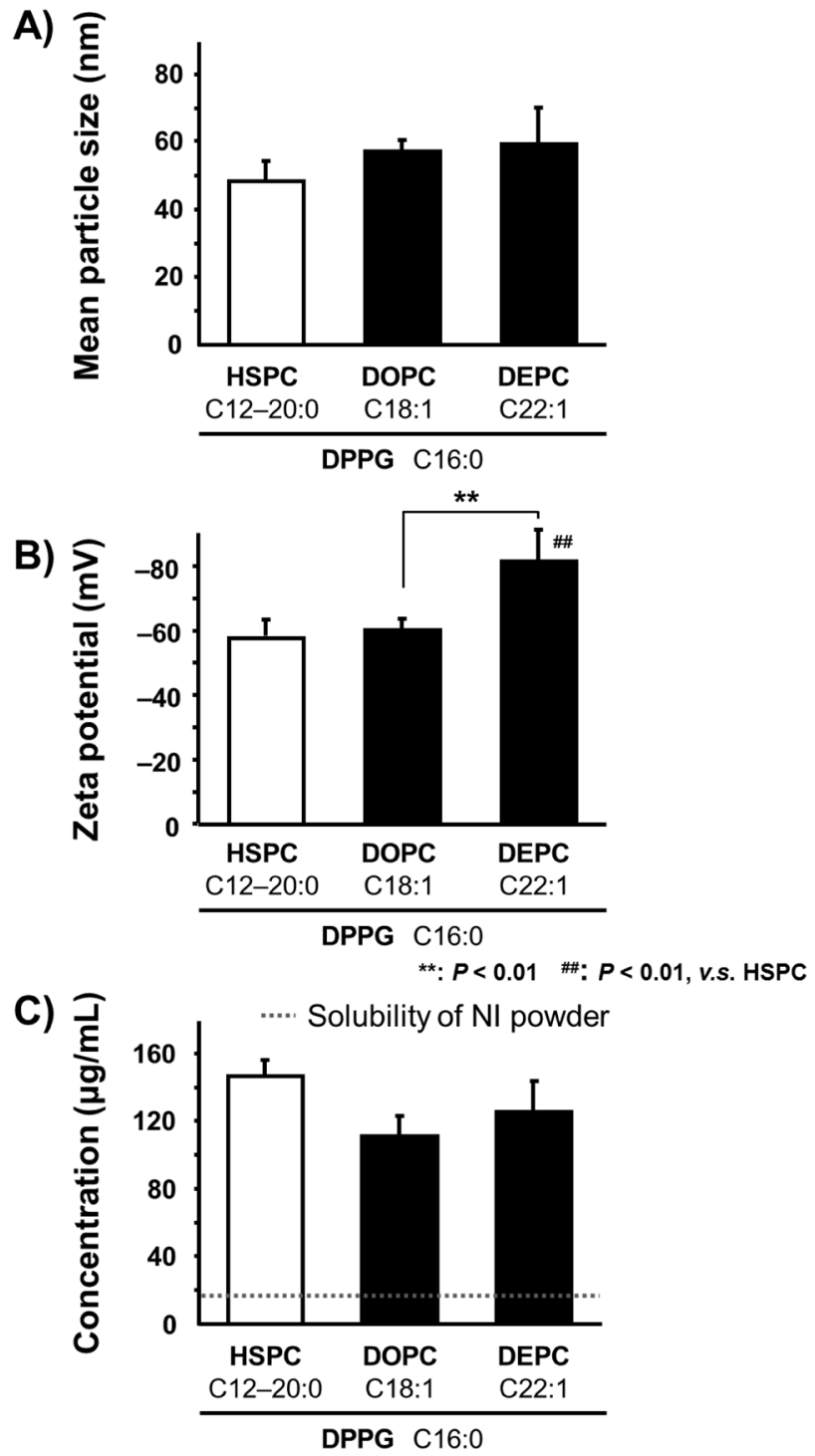

Fig. 5. Effect of an Unsaturated Bond in PC on LNs Containing DPPG (C16:0)

(A) Mean particle size, (B) zeta potential, and (C) concentration of NI. These data are the average values obtained from three experiments $( \pm$ S.D.).

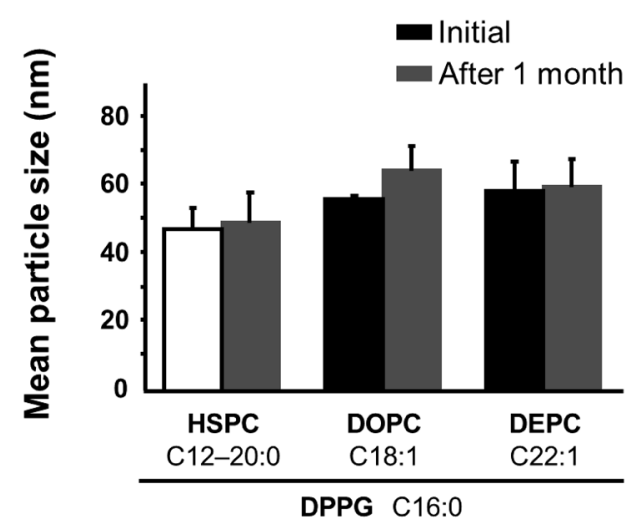

Fig. 6. Stability of LN Suspensions

These data are the average values obtained from three experiments ( \pm S.D.).

be strongly influenced by the effect of the double bond on the formation of the lipid membrane. Kučerka et al. reported that unsaturated phospholipids were not greatly affected by the alkyl chain length because the cis-double bond perturbed the hydrocarbon chain packing. ${ }^{13)}$ Therefore, LNs prepared using DOPC and DEPC would have similar mean particle sizes. Unsaturated DOPC LNs had a high drug concentration (Fig. 5C) compared with the corresponding saturated DSPC LNs with a similar alkyl chain length (Fig. 2C), although they had similar mean particle sizes. This tendency might be related to drug entrapment ability. Because unsaturated DOPC LNs have a high drug entrapment ability, the amount of NI that became entrapped in the LNs and passed through the filter was high, resulting in a high drug concentration.

Effect of the Alkyl Chain Length of PG Finally, we prepared LNs consisting of HSPC/distearoylphosphatidylglycerol (DSPG) and DSPC/DSPG. The alkyl chain of DSPG (18:0) is longer than DPPG (16:0) used previously. LNs prepared with HSPC/DSPG and DSPC/DSPG had mean particle sizes of 78 and $72 \mathrm{~nm}$, respectively (Fig. 7A). Zeta potential of LNs using HSPC/DSPG and DSPC/DSPG were -54 and $-50 \mathrm{mV}$, respectively (Fig. 7B). There were no significant differences. The drug entrapment efficiencies were 98.3 and $97.9 \%$ for LNs with HSPC/DSPG and DSPC/DSPG, respectively. The drug concentrations in LN suspensions using HSPC/DSPG and DSPC/DSPG were 51.3 and $79.1 \mu \mathrm{g} \mathrm{mL}^{-1}$, respectively (Fig. 7C). NI solubility also improved 3-4-fold in LNs prepared using DSPG as the PG. Particle size increased, and drug concentration decreased, as the alkyl chain length of the saturated PG increased, similar to the effect observed for the saturated PC. There were significant differences in both mean particle size and drug concentration between the LNs with DPPG and DSPG.

The observed trend between increased particle size and decreased drug concentration can be explained by the similar reasons as mentioned in the alkyl chain length of PC. Additionally, in this study, although the amount of PG in LNs that consisted of $\mathrm{PC}$ and $\mathrm{PG}(\mathrm{PC}: \mathrm{PG}=5: 1)$ was comparatively small, the alkyl chain length of the PG affected the physicochemical properties of the LNs, such as particle size and drug concentration (Fig. 7), rather than the alkyl chain length of the PC (Fig. 2). This tendency might be explained by differences in the structures of PG and PC. The head group volume of PG is smaller than PC and PG has repulsive electrostatic interactions. These differences result in the surface area per lipid for PG being larger than that of the corresponding PC. ${ }^{14)}$ In this study, the molecular weight of PG in the LNs was one sixth that of PC. However, the effective surface area of lipid molecules in the LNs may increase, which affects properties, such as particle size. Therefore, we consider that the effect of the alkyl chain length of PG on the mean particle size was greater than its effect on drug concentration.

\section{Conclusion}

In the range of particle sizes obtained, phospholipids having various types of alkyl chain did not affect the properties of the LNs, such as solubility and absorption. ${ }^{8)}$ From these results, it appears that although the properties of the LNs showed some variation with different phospholipids alkyl side chain lengths, overall, particles could be prepared using many kinds of phospholipids. This method shows promise for enhancing the solubility of poorly water-soluble drugs. 
A)

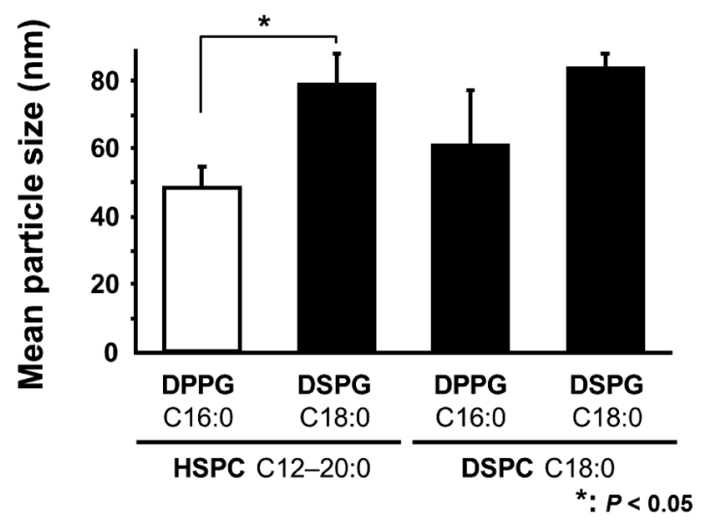

B)

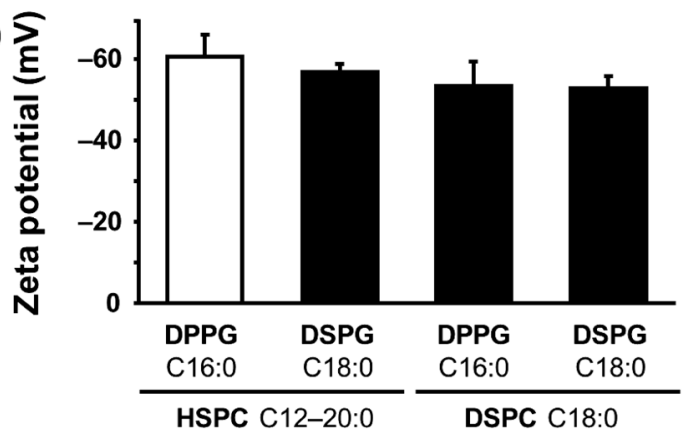

C)

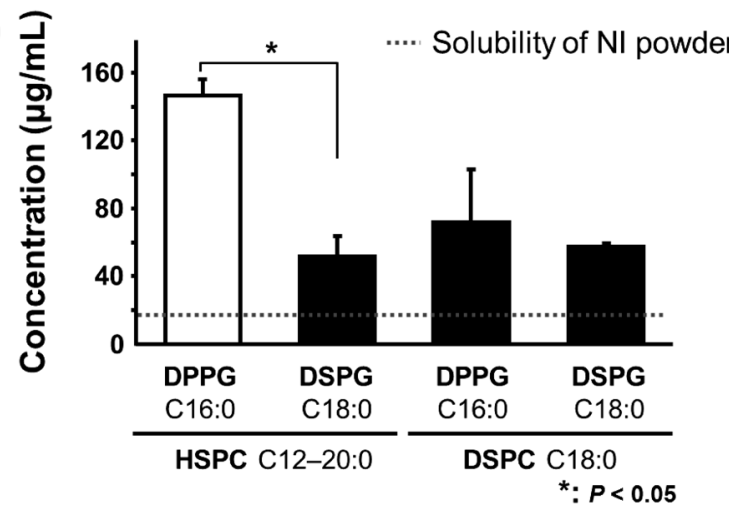

Fig. 7. Effect of the Alkyl Chain Length of PG on LNs Containing HSPC (C12-20:0) or DSPC (C18:0)

(A) Mean particle size, (B) zeta potential, and (C) concentration of NI. These data are the average values obtained from three experiments $( \pm$ S.D.).

\section{Experimental}

Materials NI, DSPC, DOPC, DEPC and DSPG were provided by Nippon Fine Chemical Co., Ltd. (Osaka, Japan). HSPC $\quad\left(\right.$ COATSOME $\left.^{\circledR} \quad \mathrm{NC}-21\right)$, DMPC $\quad$ COATSOME $^{\circledR}$ MG-4040), DPPC (COATSOME ${ }^{\circledR}$ MG-6060), and DPPG $\left(\right.$ COATSOME $^{\circledR}$ MG-6060LS) were purchased from Nippon Oil and Fats Co., Ltd. (Tokyo, Japan). Methanol (HPLC grade) and formic acid (HPLC grade) were purchased from Wako Pure Chemical Industries, Ltd. (Osaka, Japan). The membrane filters [pore size: $0.20 \mu \mathrm{m}$, polytetrafluoroethylene or cellulose acetate] were purchased from Toyo Roshi Kaisha Ltd. (Tokyo, Japan). All reagents were of the highest grade commercially available and all solutions were prepared using deionized distilled water.

Preparation of NI-LN Suspensions NI $(40 \mathrm{mg})$ and lipid (1000 mg; $P C: P G=5: 1$ molar ratio) were physically mixed in a mortar for $5 \mathrm{~min}$. The mixture was then co-ground by a roll mill (R3-1R; Kodaira Seisakusho Co., Ltd., Tokyo, Japan) for
$5 \mathrm{~min}$. The resultant roll mixture was dispersed in $200 \mathrm{~mL}$ of deionized distilled water and premixed using a Speed Stabilizer $^{\circledR}$ (Kinematica Co., Lucerne, Switzerland) at $9000 \mathrm{rpm}$ for $10 \mathrm{~min}$. Then, the coarse dispersions were subjected to high pressure homogenization (Microfluidizer ${ }^{\circledR}, \mathrm{M} 110-\mathrm{E} / \mathrm{H}$; Microfluidics, Co., Newton, MA, U.S.A.) at $175 \mathrm{MPa}$ with pass cycles of 100. Previously, when the NI-LN suspensions containing $20,25,30,40,50$, or $80 \mathrm{mg}$ of NI and $1000 \mathrm{mg}$ of lipid mixture (HSPC and DPPG) were prepared, deposited NI crystal was observed within $12 \mathrm{~h}$ after preparation in the LN suspensions containing more than $50 \mathrm{mg}$ of NI. Therefore, we have fixed the molar ratio of drug and lipid mixture in this study.

Evaluation of the Physical Properties of NI-LN Suspensions The mean particle size and zeta potential of prepared NI-LN suspensions were measured using an electrophoretic light scattering photometer (ELS-8000; Otsuka Electronics Co., Ltd., Osaka, Japan, for the mean particle size, Zetasizer nano ZS, Sysmex Co., Ltd., for zeta potential) at room temperature.

The Concentration and the Entrapment Efficiency of NI in LN Suspensions NI-LN suspensions were filtered through a $0.2-\mu \mathrm{m}$ membrane filter. Filtered LNs were defined as the soluble state in this study. The concentration of NI was determined as follows: aliquots of $200-\mu \mathrm{L}$ NI-LN suspensions were dissolved and diluted appropriately with methanol. The resulting solution was analyzed by HPLC (LC-2010CHT; Shimadzu, Kyoto, Japan). The analytical column was Cadenza CD-C18, $3 \mu \mathrm{m}, 4.6 \mathrm{~mm} \times 150 \mathrm{~mm}$ (Imtakt Corp., Kyoto, Japan). The detector was a UV detector (wavelength: $230 \mathrm{~nm}$ ), column temperature was $40^{\circ} \mathrm{C}$, the mobile phase was methanol/ water $=5(\mathrm{v} / \mathrm{v})$, and flow rate was $0.6 \mathrm{~mL} / \mathrm{min}$. Encapsulation efficiency of NI in the LN suspensions was calculated by determining the amount of free drug using ultrafiltration. NI-LN suspensions $(500 \mu \mathrm{L})$ were placed on an ultrafilter of Amicon $^{\circledR}$ Ultra-0.5 Centrifugal Filter Devices (50 K device 50000 MNWL; Merck Millipore Ltd., Billerica, MA, U.S.A.) in a centrifuge tube and centrifuged at $20817 \times \boldsymbol{g}$ at $4^{\circ} \mathrm{C}$ for $10 \mathrm{~min}$. The ultrafiltrate containing the unencapsulated drug was analyzed by HPLC. The entrapment efficiency of NI in LN suspensions was calculated as follows: entrapment efficiency $(\%)=($ total drug content - unencapsulated drug content $) /$ total drug content $\times 100$.

Stability Studies of NI-LN Suspensions The physical stability of the LN suspensions was evaluated as previously described. ${ }^{15)}$ Briefly, the NI-LN suspensions were kept in a closed clear glass beaker and stored at $4^{\circ} \mathrm{C}$. At $30 \mathrm{~d}$ after preparation, the mean particle size was measured as noted above.

Statistics The Student's $t$-test was used to assess the significance of the differences among the various groups. Results with $p<0.01$ or $p<0.05$ were considered to be statistically significant.

Conflict of Interest The authors declare no conflict of interest.

\section{References}

1) Mehnert W., Mäder K., Adv. Drug Deliv. Rev., 64, 83-101 (2012). 2) Law D., Wang W., Schmitt E. A., Qiu Y., Krill S. L., Fort J. J., J. Pharm. Sci., 92, 505-515 (2003).

3) Jinno J., Kamada N., Miyake M., Yamada K., Mukai T., Odomi M., 
Toguchi H., Liversidge G. G., Higaki K., Kimura T., J. Controlled Release, 111, 56-64 (2006).

4) Kamiya S., Yamada M., Kurita T., Miyagishima A., Arakawa M., Sonobe T., Int. J. Pharm., 354, 242-247 (2008).

5) Funakoshi Y., Iwao Y., Noguchi S., Itai S., Int. J. Pharm., 451, 92-94 (2013)

6) Ohshima H., Miyagishima A., Kurita T., Makino Y., Iwao Y., Sonobe T., Itai S., Int. J. Pharm., 377, 180-184 (2009).

7) Barman R. K., Iwao Y., Funakoshi Y., Ranneh A. H., Noguchi S., Wahed M. I. I., Itai S., Chem. Pharm. Bull., 62, 399-406 (2014).

8) Barman R. K., Iwao Y., Islam M. R., Funakoshi Y., Noguchi S., Wahed M. I. I., Itai S., Pharmacology \& Pharmacy, 5, 455-461 (2014).

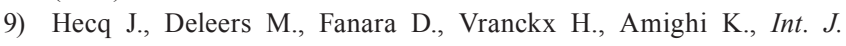

Pharm., 299, 167-177 (2005).

10) Mohammed A. R., Weston N., Coombes A. G. A., Fitzgerald M., Perrie Y., Int. J. Pharm., 285, 23-34 (2004).

11) Chen C. Y. C., J. Taiwan Inst. Eng., 40, 380-386 (2009).

12) Verma S., Kumar S., Gokhale R., Burgess J. D., Int. J. Pharm., 406, 145-152 (2011).

13) Kučerka N., Nieh M. P., Katsaras J., Biochim. Biophys. Acta, 1808, 2761-2771 (2011).

14) Pan J., Heberle F. A., Tristram-Nagle S., Szymanski M., Koepfinger M., Katsaras J., Kučerka N., Biochim. Biophys. Acta, 1818, 21352148 (2012)

15) Kamiya S., Nozawa Y., Miyagishima A., Kurita T., Sadzuka Y., Sonobe T., Chem. Pharm. Bull., 54, 181-184 (2006). 